Pacific Journal of Mathematics

SINGULARITIES IN A VARIATIONAL PROBLEM WITH AN
INEQUALITY 


\section{SINGULARITIES IN A VARIATIONAL PROBLEM WITH AN INEQUALITY}

\section{Boris Garfinkel AND Gregory T. McAllister}

The subject of the paper is the variational problem of Lagrange with an inequality in the form $(a) \phi(x, y) \geqq 0$ or (b) $\phi\left(x, y, y^{\prime}\right) \geqq 0$. The question of existence and uniqueness of the continuation of a minimizing arc is investigated at points of the boundary $\phi=0$. Various phenomena, including splitting of extremals, dead-end, entry into, and exit from the boundary, are treated and the conditions for their occurrence are derived. The nature of the continuation is related to the "index" associated with an extremal.

An appendix extends the results to a control problem of the Mayer type.

In the variational problem of Lagrange with the inequality (a) $\phi(x, y) \geqq 0$ or (b) $\phi\left(x, y, y^{\prime}\right) \geqq 0$, case (a) has been treated by Bolza [3] and Mancil [6], and case (b) by Valentine [8]. Despite the relative antiquity of the problem several questions have remained unresolved.

A difficulty arises when an extremal of the problem has no unique continuation. We distinguish continuations in the region $\phi>0$, and continuations in the boundary $\phi=0$. Let the type of continuation not be specified a priori, and let $H$ denote the corresponding Hilbert determinant of the composite arc. As will be shown, in case (a)

$$
H=\left|\begin{array}{cc}
f_{y^{\prime} y^{\prime}} & -\phi_{y} \\
0 & \phi
\end{array}\right|
$$

and $H=0$ if and only if $\phi=0$; in case (b)

$$
H=\left|\begin{array}{cc}
F_{y^{\prime} y^{\prime}} & \phi_{y^{\prime}} \\
\lambda \phi_{y^{\prime}} & \phi
\end{array}\right|
$$

where $\lambda$ is a Lagrange multiplier and $F \equiv f+\lambda \phi$, and $H=0$ if and only if $\phi=\lambda=0$. Since a solution generally contains points of the boundary, clearly the singularities defined by $H=0$ deserve attention. For not only do they arise in practical problems, as noted by Garfinkel [5] and others, but they are also of intrinsic mathematical interest.

A systematic treatment of such singularities is undertaken here. Various phenomena, including splitting of extremals, dead-end, entry into, and exit from the boundary, will be treated and the conditions for their occurrence derived. It will be shown that case (a) exhibits the splitting and the dead-end, in contrast case (b), where a unique

Received May 6, 1964. 
continuation exists.

Let $\widetilde{R}$ be a set of points $(x, y, w)$ for which $x_{1} \leqq x \leqq x_{2}$ and $\phi(x, y, w) \geqq 0$. Assume that functions $f(x, y, w)$ and $\phi(x, y, w)$ have continuous derivatives of order $r+2$ in $\widetilde{R}$. An admissible arc $y(x)$ has a piecewise continuous first derivative, with a possible discontinuity in $y^{\prime}$, i.e. $y(x)$ is of class $\left(C^{1}, D^{1}\right)$ in the notation of Bolza, with $\left(x, y, y^{\prime}\right)$ in $\widetilde{R}$, and its end-points $\left(x_{1}, y\left(x_{1}\right)\right),\left(x_{2}, y\left(x_{2}\right)\right)$ fixed. In the class of admissible arcs, we seek a $y(x)$ that minimizes the integral

$$
J=\int_{x_{1}}^{x_{2}} f\left(x, y, y^{\prime}\right) d x
$$

Within this general formulation, we shall investigate the singularities that arise on $\phi=0$.

The attack on the problem begins in $\S 2$ with a discussion of the necessary and sufficient conditions I-IV of the calculus of variations, which are applied in $\S \S 3$ and 5 to the point of junction of the region and the boundary subarcs. Finally, the singularities in question are treated in $\S \S 4$ and 6 .

A restatement of the known and relevant theory, designed to unify the subject and to provide a sufficient background for the current development, is incorporated in $\S \S 2,3$, and 5 .

2. Necessary and Sufficient Conditions. Let $R$ and $B$ be the subsets of $\widetilde{R}$ for which $\phi>0$ and $\phi=0$, respectively. Subarcs in $R$ and $B$ will be referred to as region, or $R$-subarcs, and boundary, or $B$-subarcs, respectively, and may be abbreviated as $\bar{R}$ and $\bar{B}$.

An admissible curve $y(x)$ is generally compounded of $R$-subarcs, on which $\phi>0$ and $\lambda=0$, and of $B$-subarcs, on which $\phi=0$ and $\lambda \neq 0$ is admitted. Let such a composite curve lie in $R$ for $x_{1} \leqq x<\xi$ and in $B$ for $\xi \leqq x \leqq x_{2}$, and have no corners except possibly at the junction $x=\xi$.

Construct the function $F=f+\lambda \phi$, where the Lagrange multiplier $\lambda(x)$ satisfies $\lambda \phi=0$. For the sake of notational compactness, define the $(n+1)-$ vectors $z=(x, y)$ and $p\left(x, y, y^{\prime}\right)$ :

$$
p_{0}=F-y^{\prime} \cdot F_{y^{\prime}}, \quad p_{i}=F_{y_{i}^{\prime}},
$$$$
i=1, \cdots n,
$$

where the dot denotes the inner product of vectors. Then condition I, $d J \geqq 0$ implies the Euler condition

$$
\frac{d}{d x} F_{y^{\prime}}=F_{y}, \quad \lambda \phi=0
$$


holding between corners; the Weierstrass-Erdmann condition

$$
\left.\Delta p \cdot d z\right|_{\xi}=0,
$$

for all $d z$ satisfying $\mathrm{d} \phi=0$ at $x=\xi$, with $\Delta p=p_{+}-p_{-}$denoting a jump in $p(x)$; the convexity condition

$$
\lambda(x) \leqq 0 \text {. }
$$

The latter was derived by Bolza for case (a), and by Valentine for case (b). We shall use the term extremaloid [7] to describe arcs that satisfy I $a, I b$, and I c.

The necessary conditions for a minimum also include condition II of Weierstrass, condition III of Legendre, and an appropriate modification of condition IV of Jacobi. These conditions and the sufficient conditions $\mathrm{II}_{N}^{\prime}[1]$, III', and IV' $^{\prime}$ are well known, and the last two can be found in Valentine. For the purposes of our paper, we shall impose the following condition III", which is somewhat stronger than III':

$F_{y^{\prime} y^{\prime}}$ is a positive-definite matrix for all $(x, y, \lambda)$ on the extremal and for all $y^{\prime}$.

3. Non-Singular Points in Case (a). The question of existence and uniqueness of an extremal continuation at points that are neither corners nor junctions has been settled by the Hilbert Differentiability Theorem [2]. On $\bar{R}$ and on $\bar{B}$, the hypothesis of the theorem is the nonvanishing of the Hilbert determinants; i.e.,

$$
\begin{aligned}
& H_{1}=\left|f_{y^{\prime} y^{\prime}}\right| \neq 0, \\
& H_{2}=\phi_{y^{\prime}} \cdot \widehat{f}_{y^{\prime} y^{\prime}} \cdot \phi_{y} \neq 0,
\end{aligned}
$$

where the symbol $\hat{\Lambda}$ denotes the adjoint of a matrix. The condition is assured by III" and by the assumed normality $\phi_{y} \neq 0$ on the extremal. Then the theorem implies that $y^{\prime \prime}$ and $\lambda$ exist and are in $C^{r}$, and that the Euler equations have a unique solution.

The behavior of extremals at junctions has been investigated with the aid of the corner condition. Since $\phi_{y^{\prime}} \equiv 0, d z(\xi)$ in (4) must satisfy $d \phi=\phi_{z} \cdot d z=0$ and $\Delta p \cdot d z=0$, which implies

$$
\Delta p=\kappa \phi_{z} \text { at } x=\xi,
$$

where $\kappa$ is a constant of proportionality. From the system (7), of $n+1$ equations, the $n+1$ unknowns, $y_{+}^{\prime}, \kappa$ are to be determined. At a corner, the $E$-function with arguments $y^{\prime}=y_{-}^{\prime}$ and $Y^{\prime}=y_{+}^{\prime}$ is given by the expression 


$$
E\left(y_{-}^{\prime}, y_{+}^{\prime}\right)=\Delta f-\left(f_{y_{-}^{\prime}}\right) \cdot \Delta y^{\prime}=\kappa \phi_{+}^{\prime} .
$$

For entry into the boundary, $\phi_{+}^{\prime}=0$ and (8) implies $E=0$. Since $f$ is in $C^{r+2}, f_{y^{\prime} y^{\prime}}$ exists and is continuous. By the Mean Value Theorem applied to (8), there exists a number $\theta$ in the interval $(0,1)$ such that

$$
E\left(y_{-}^{\prime}, y_{+}^{\prime}\right)=\Delta y^{\prime} \cdot f_{y^{\prime} y^{\prime}}\left(y_{-}^{\prime}+\theta \Delta y^{\prime}\right) \cdot \Delta y^{\prime} .
$$

Condition III" and $E=0$ then imply

$$
\Delta y^{\prime}=0, \quad \Delta \phi^{\prime}=0 .
$$

The corner condition is then trivially satisfied with $\Delta p=0, \kappa=0$, and the incident extremal must be tangent [4] to the boundary $\phi=0$. It may be pointed out that, in order to establish (10), the positivedefiniteness of $f_{y^{\prime} y^{\prime}}$ is required only for $\theta$ in $(0,1)$. Hence condition III" is stronger than necessary.

Entry is thus subject to the conditions

$$
\phi=\phi^{\prime}=0 \text { at } x=\xi,
$$

connecting the two unknowns $\xi$ and $\alpha_{k}$, where $\alpha_{k}$ is suitably chosen among the $n$ parameters of the family $y(x, \alpha)$. It is noteworthy that a degree of freedom is lost upon entry, with the $n$-parameter family of $R$-extremals degenerating into an $(n-1)$-parameter family of $B$-extremals, with $\alpha_{k}$ fixed.

At a point of exit from the boundary, the tangency condition (10) is established by symmetry. Thus, while entry is limited to $R$-arcs that are tangent to the boundary, exit from the boundary is unrestricted provided $\lambda(\xi)<0$. The variable $\xi$ thus becomes a family parameter of the emergent $R$-arcs, replacing the lost parameter $\alpha_{k}$, and the degree of freedom lost on entry is restored on exit. While (10) is necessary for both transitions, it is by no means sufficient, as will be shown in $\S 4$.

Since $y^{\prime}$ is continuous, and since $y^{\prime \prime}$ and $\lambda$ are generally discontinuous at junctions, it follows from the Hilbert Theorem that $y^{\prime \prime}(x)$ and $\lambda(x)$ are in $\left(C^{r}, D^{o}\right)$.

4. Singularities in Case (a). On a composite arc, the system (3) is linear in the unknowns $y^{\prime \prime}, \lambda$ and has the Hilbert determinant

$$
H=\left|\begin{array}{cc}
f_{y^{\prime} y^{\prime}} & -\phi_{y} \\
0 & \phi
\end{array}\right|=\phi\left|f_{y^{\prime} y^{\prime}}\right| \text {. }
$$

A unique solution exists if and only if $H \neq 0$ at $x=\xi$. Accordingly, we define singular points by the condition $H=0$, which implies $\phi=0$ in virtue of III'. Singularities therefore occur on $\phi=0$ in transitions 
classified below:

Table 1. Types of Transition

\begin{tabular}{l|c|l|l}
\hline \multicolumn{1}{c|}{ Type } & Symbol & Name & Remarks \\
\cline { 1 - 2 } Region-boundary & $\mathrm{RB}$ & entry & nontrivial \\
Boundary-region & $\mathrm{BR}$ & exit & nontrivial \\
Region-region & $\mathrm{RR}$ & nonentry & trivial \\
Boundary-boundary & $\mathrm{BB}$ & nonexit & trivial \\
\hline
\end{tabular}

Let us define $p$ as the least integer such that $p \leqq r$ and $y_{+}^{(p+2)}(\xi) \neq y_{-}^{(p+2)}(\xi)$ in a nontrivial transition. The exceptional case where $p$ does not exist is treated in Appendix 1.

Lemma (a). If $\phi_{y^{\prime}} \equiv 0, \phi_{y} \neq 0$ on the extremal, and III" holds, then at a singular point $x=\xi$

$$
\Delta \phi^{(p+2)} \Delta \lambda^{(p)} \geqq 0,
$$

where the equality holds only for the trivial transitions, with both factors vanishing.

Proof. For a nontrivial transition an application of the jump operator $\Delta$ to the Euler equation in (3) yields

$$
f_{y^{\prime} y^{\prime}} \cdot \Delta y^{\prime \prime}(\xi)=\phi_{y} \Delta \lambda(\xi),
$$

in view of the continuity of $y$ and $y^{\prime}$. It follows by III" and normality that both $\Delta y^{\prime \prime}$ and $\Delta \lambda$ vanish or do not vanish simultaneously. The definition of $p$ and the successive differentiation of the Euler equation leads to

$$
f_{y^{\prime} y^{\prime}} \cdot \Delta y^{(p+2)}(\xi)=\phi_{y} \Delta \lambda^{(p)}(\xi) \neq 0 .
$$

By analogous reasoning, the application of the $\Delta$-operator to the function $\phi^{\prime \prime}$ yields

$$
\phi_{y} \cdot \Delta y^{\prime \prime}(\xi)=\Delta \phi^{\prime \prime}(\xi),
$$

and

$$
\phi_{y} \cdot \Delta y^{(p+2)}(\xi)=\Delta \phi^{(p+2)}(\xi) \text {. }
$$

The elimination of $\Delta y^{(p+2)}$ from (17) and (15) now leads to

$$
\Delta \phi^{(p+2)}=\left(\phi_{y} \cdot f_{y^{\prime} y^{\prime}}^{-1} \cdot \phi_{y}\right) \Delta \lambda^{(p)}
$$

and, finally, by III" and normality, the conclusion follows.

CoRollary (a). Under the same hypothesis, 


$$
\phi_{+}^{(p+2)} \lambda_{+}^{(p)}<0,
$$

where the two factors belong to the $\bar{R}$ and the $\bar{B}$ continuations respectively.

Proof. Two cases occur, which shall be referred to as $(R)$ and $(B)$.

Case $(R)$. If $\xi$ belongs to a $R$-arc, the $R B$ and the $R R$ transitions must be considered. For the $R B$ transition $\lambda(x)=0, \lambda_{-(p)}^{(\xi)}=0$ for $x \leqq \xi$, and $\phi(x)=0, \phi_{+}^{(p+2)}(\xi)=0$ for $x \geqq \xi$. Hence (13) becomes $\phi^{(p+2)} \lambda_{+}^{(p)}<0$, where the two factors are the lowest non-vanishing derivatives at $\xi$. For the $R R$ transition all the existing derivatives of $y(x)$ are continuous at $\xi$, so that $\phi_{-}^{(p+2)}=\phi_{+}^{(p+2)}$. Since $\phi_{-}$refers to the same $R$-arc, the last two equations imply (19).

Case $(B)$. If $\xi$ belongs to a $R$-arc, the $B R$ and the $B B$ transitions must be considered. Analogous reasoning shows that $\phi_{+}^{(p+2)} \lambda_{-}^{(p)}<0$ and $\lambda_{-}^{(p)}=\lambda_{+}^{(p)}$, again leading to (19).

We shall now inquire whether the two continuations indicated in (19) meet both the requirements $\phi \geqq 0$ and $\lambda \leqq 0$. The question is settled by

THEOREM (a). If the hypothesis of Lemma (a) holds, then the extremal has either two continuations or none:

(1) for $p$ even, there exists a continuation in the region and a continuation in the boundary;

(2) for $p$ odd, there is no continuation.

Proof. Case $(R)$. If $\xi$ belongs to a $R$-arc, the dominant terms of the Taylor series expansion of $\lambda(x)$ and $\phi(x)$ about $\xi$ in powers of $\varepsilon=|x-\xi|$ are given by:

$$
\begin{aligned}
& \lambda(\xi+\varepsilon)=\varepsilon^{p} \lambda_{+}^{(p)}(\xi) / p !+\cdots, \\
& \phi(\xi \pm \varepsilon)=( \pm 1)^{p} \varepsilon^{p+2} \phi^{(p+2)}(\xi) /(p+2) !+\cdots,
\end{aligned}
$$

for the $R B$ and the $R R$ continuations respectively. Since the given $R$-arc is admissible, $\phi(\xi-\varepsilon)>0$. Then (20) and (19) imply exactly two possibilities:

(1) $p$ is even: $\phi^{\left(p_{+2}\right)}>0, \phi(\xi+\varepsilon)>0, \quad \lambda_{+}^{(p)}<0, \quad \lambda(\xi+\varepsilon)<0$. The $R B$ and the $R R$ transitions satisty the respective requirements $\lambda(\xi+\varepsilon)<0$ and $\phi(\xi+\varepsilon)>0$. Both continuations being possible, an extremaloid arc tangent to the boundary at $x=\xi$ splits off a boundary subarc.

(2) $p$ is odd: $\phi^{(p+2)}<0, \quad \phi(\xi+\varepsilon)<0, \quad \lambda_{+}^{(p)}>0, \quad \lambda(\xi+\varepsilon)>0$. Both transitions violate the respective requirements, and neither con- 
tinuation is possible; the extremaloid thus comes to a dead-end.

Case (B). If $\xi$ belongs to a $B$-arc, (20) is replaced by

$$
\begin{aligned}
& \phi(\xi+\varepsilon)=\varepsilon^{p+2} \phi_{+}^{(p+2)}(\xi) /(p+2) !+\cdots, \\
& \lambda(\xi \pm \varepsilon)=( \pm 1)^{p} \varepsilon^{p} \lambda^{(p)}(\xi) / p !+\cdots
\end{aligned}
$$

Since the given $B$-arc satisfies $\lambda(\xi-\varepsilon)<0$, (21) and (19) again imply exactly two possibilities:

(1) $p$ is even: $\lambda^{(p)}<0, \quad \lambda(\xi+\varepsilon)<0, \quad \phi_{+}^{(p+2)}>0, \quad \phi(\xi+\varepsilon)>0$. Both continuations being possible, a boundary extremaloid splits off, at $x=\xi$, a tangent subarc.

(2) $p$ is odd: $\lambda^{(p)}>0, \lambda(\xi+\varepsilon)>0, \phi_{+}^{(p+2)}<0, \phi(\xi+\varepsilon)<0$. Neither continuation being possible, the extremaloid comes to a deadend.

An illustration of the theory is furnished by the following example. Consider a boundary extremal subarc at $\xi=0$ with

$$
f=\sqrt{1+y^{\prime 2}}, \quad \phi=y-x^{3} .
$$

Then $y(x)=x^{3}, y^{\prime}=3 x^{2}, y^{\prime \prime}=6 x, y^{\prime \prime \prime}=6$. From $(3)$

$$
\begin{aligned}
\lambda(x) & =\left(\frac{d}{d x} f_{y^{\prime}}-f_{y}\right) / \phi_{y} \\
& =6 x\left(1+9 x^{4}\right)^{-3 / 2} .
\end{aligned}
$$

Hence $\lambda(\xi)=0, \lambda^{\prime}(\xi)=6$, and $p=1$. Since $p$ is odd, case (2) applies, with the diagnosis of a dead-end. A string stretched along a convex boundary $\phi=0$ provides a physical interpretation of the fact that a geodesic $y(x)$ has no continuation beyond a point of inflection, where $y^{\prime \prime}(\xi)=0$ and $y^{\prime \prime \prime}(\xi)>0$.

5. Nonsingular Points in Case (b). As in $\S 3$, an extremal has a unique continuation between junctions. A significant change in the analysis leading to (6) is the replacement of $\left(f, \lambda, \phi_{y}, \phi^{\prime \prime}\right)$ by $\left(F, \lambda^{\prime}, \phi_{y^{\prime}}, \phi^{\prime}\right)$ respectively, with the assumed normality $\phi_{y^{\prime}} \neq 0$ on the extremal. There follows the conclusion that the Euler equations have a unique solution $y^{\prime \prime}$ and $\lambda^{\prime}$.

Since $\phi_{y^{\prime}} \neq 0, d z$ in (4) is arbitrary and the corner condition becomes

$$
\Delta p(\xi)=0,
$$

which furnishes $n+1$ equations from which the $n+1$ unknowns $y_{+}^{\prime}$, $\lambda_{+}$are to be determined. Hence

$$
E\left(y_{-}^{\prime}, y_{+}^{\prime}\right)=0
$$


by reasoning analogous to that in $\S 3$. An assumption on $F$ like the one in (9) finally implies

$$
\Delta y^{\prime}=0 \text {. }
$$

From (24) and (23) it follows that $\lambda_{+}=0$; since $\lambda_{-}=0, \lambda$ is continuous at $\xi$, and

$$
\Delta \lambda=0
$$

Entry is thus subject to the conditions

$$
\phi=0, \quad \Delta y^{\prime}=0, \quad \Delta \lambda=0 .
$$

By symmetry, the conditions

$$
\lambda=0, \quad \Delta y^{\prime}=0, \quad \Delta \lambda=0
$$

hold for exit. In both transitions, the unknowns $\xi, y_{+}^{\prime}, \lambda_{+}$are completely determined. That these conditions are not sufficient will be shown in $\S 6$.

Since $y^{\prime}$ and $\lambda$ are continuous, and since $y^{\prime \prime}$ and $\lambda^{\prime}$ are generally discontinuous at junctions, it follows from the Hilbert Theorem that $y^{\prime \prime}(x)$ and $\lambda^{\prime}(x)$ are in $\left(C^{r}, D^{o}\right)$.

6. Singularities in Case (b). For a composite arc the system (3) is replaced by

$$
\frac{d}{d x} F_{y^{\prime}}=F_{y}, \quad \frac{d}{d x}(\lambda \phi)=0
$$

which is linear in $y^{\prime \prime}, \lambda^{\prime}$ and has the Hilbert determinant

$$
H=\left|\begin{array}{cc}
F_{y^{\prime} y^{\prime}} & \phi_{y^{\prime}} \\
\lambda \phi_{y^{\prime}} & \phi
\end{array}\right|=\phi\left|F_{y^{\prime} y^{\prime}}\right|-\lambda \phi_{y^{\prime}} \cdot \hat{F}_{y^{\prime} y^{\prime}} \cdot \phi_{y^{\prime}} \bullet
$$

From III', normality, and $\lambda \phi=0$ there follows $H=0$ if and only if $\phi=\lambda=0$.

Lemma (b). If $\phi_{y^{\prime}} \neq 0$ on the extremal, and III" holds, then at $a$ singular point $x=\xi$

$$
\Delta \phi^{(p+1)} \Delta \lambda^{(p+1)} \leqq 0,
$$

where the equality holds only for the trivial transitions, with both factors vanishing.

The proof proceeds as in $\S 4$, Lemma (a), with the replacement of $\left(\lambda, \phi_{y}, \phi^{\prime \prime}\right)$ by $\left(-\lambda^{\prime}, \phi_{y^{\prime}}, \phi^{\prime}\right)$ in $(14-18)$. 
CoRollary (b). Under the same hypothesis,

$$
\phi_{+}^{(p+1)} \lambda_{+}^{(p+1)}>0,
$$

where the two factors belong to the $\bar{R}$ and the $\bar{B}$ continuations, respectively.

The proof proceeds as in $\S 4$, Corollary (a), with the replacement of $\phi^{(p+2)}, \lambda^{(p)}$ by $\phi^{(p+1)}, \lambda^{(p+1)}$, respectively.

THEOREM (b). If the hypothesis of Lemma (b) holds, then the extremal has a unique continuation:

(1) for $p+1$ even, a $R$-arc continues in $R$, and a $B$-arc continues in $B$;

(2) for $p+1$ odd, a $R$-arc continues in $B$, and a $B$-arc continues in $R$.

Proof. Case (R). Equation (20) is replaced by

$$
\begin{aligned}
& \lambda(\xi+\varepsilon)=\varepsilon^{p+1} \lambda_{+}^{(p+1)}(\xi) /(p+1) !+\cdots, \\
& \phi(\xi \pm \varepsilon)=( \pm 1)^{p_{+1}} \varepsilon^{p_{+1}} \phi^{\left(p_{+1}\right)}(\xi) /(p+1) !+\cdots .
\end{aligned}
$$

Then (32), (31) and $\phi(\xi-\varepsilon)>0$ imply either (1) or (2):

(1) $p+1$ is even: the quantities $\phi^{(p+1)}, \phi(\xi+\varepsilon), \lambda_{+}^{(p+1)}, \lambda(\xi+\varepsilon)$ are positive; the extremaloid continues in the region and no entry can occur.

(2) $p+1$ is odd: the same quantities are negative, so that entry is the unique extremaloid continuation.

Case $(B)$. Equation (21) is replaced by

$$
\begin{aligned}
& \phi(\xi+\varepsilon)=\varepsilon^{p_{+1}} \phi_{+}^{(p+1)}(\xi) /(p+1) !+\cdots, \\
& \lambda(\xi \pm \varepsilon)=(-1)^{p_{+1}} \varepsilon^{p_{+1}} \lambda^{(p+1)}(\xi) /(p+1) !+\cdots .
\end{aligned}
$$

Now (33), (31), and $\lambda(\xi-\varepsilon)<0$ again imply either (1) or (2):

(1) $p+1$ is even: the quantities $\lambda^{(p+1)}, \lambda(\xi+\varepsilon), \phi_{+}^{(p+1)}, \phi(\xi+\varepsilon)$ are negative; the extremaloid continues in the boundary and no exit can occur.

(2) $p+1$ is odd: the same quantities are positive, so that exit from the boundary is the unique extremaloid continuation.

7. The Index of an Extremal. Since $y^{(k+2)}(x)$ at $x=\xi_{\text {- }}$ exists for $k=0,1, \cdots, r$ and must be presumed known, the lowest nonvanishing derivative of either $\phi(x)$ or $\lambda(x)$ at $\xi_{-}$can be determined. This may be done by the repeated differentiation of

$$
\phi(x)=\phi\left(x, y(x), y^{\prime}(x)\right)
$$

if the subarc is in $R$, or of the Euler equation 


$$
\lambda^{\prime} \phi_{y^{\prime}}+\lambda\left(\frac{d}{d x} \phi_{y^{\prime}}-\phi_{y}\right)+\frac{d}{d x} f_{y^{\prime}}-f_{y}=0
$$

if the subarc is in $B$. Let $q$ be the order to the lowest nonvanishing derivative, and let the index $i(\xi)$ be defined as 0 if $q$ is even and as 1 if $q$ is odd. Clearly $i(x)=0$ almost everywhere; the exceptions are dead-end in case (a), and entry and exit in case (b).

In terms of the index $i(\xi)$, the behavior of an extremaloid can be summarized by the following

THEOREM. If $x=\xi$ is a singular point of an extremaloid, and

(a) If $\phi_{y^{\prime}} \equiv 0$, then the extremaloid either splits, undergoing both a trivial and a nontrivial transitions, or it comes to a dead-end, depending on whether the index is zero or one.

(b) If $\phi_{y^{\prime}} \neq \equiv 0$, then the extremaloid undergoes a trivial or a nontrivial transition, depending on whether the index is zero or one.

Note that this statement covers two situations not included in the proofs of $\S \S 4,6$ :

1. In case (a), if $\phi_{-}^{\prime}<0$ and $q=i=1$, a dead-end occurs, since a corner on the boundary is excluded by our assumption on $F$.

2. In case (b), if $\lambda_{-}<0$ and $q=i=0$, there is only a trivial transition, BB. If $\phi(\xi)>0$, then $i=0$, and only the trivial transition $R R$ occurs in both cases (a) and (b).

Details appear in Table 2, with $N$ denoting the number of continuations, and the word Type referring to the type of transition.

Table 2. Behavior on the Boundary

\begin{tabular}{l|c|c|c|cc|cc}
\hline$q$ & $i$ & $\phi_{-}^{(q)}$ & $\lambda_{-}^{(q)}$ & $N$ & $\begin{array}{c}\text { Case (a) } \\
\text { Type }\end{array}$ & $N$ & $\begin{array}{c}\text { Case (b) } \\
\text { Type }\end{array}$ \\
\hline even & 0 & + & 0 & 2 & RB (entry) and RR & 1 & $\mathrm{RR}$ \\
odd & 1 & - & 0 & 0 & dead-end & 1 & $\mathrm{RB}$ \\
even & 0 & 0 & - & 2 & BR (exit) and BB & 1 & $\mathrm{BB}$ \\
odd & 1 & 0 & + & 0 & dead-end & 1 & $\mathrm{BR}$ \\
\hline
\end{tabular}

The existence and uniqueness of extremaloid continuation have been determined. The case $\phi(x, y) \geqq 0$ exhibits the singularities of splitting and dead-end, in contrast to the case $\phi\left(x, y, y^{\prime}\right) \geqq 0$, where a unique continuation exists. In all cases, the nature of the continuation depends on the extremaloid index $i(\xi)$; i.e. the parity of the lowest order of the set of nonvanishing derivatives of either $\phi(x)$ or $\lambda(x)$ at a given point $x=\xi$. 
APPENDIX 1. An exceptional situation arises if the index $i(\xi)$ does not exist because all the existing derivatives of $y(x)$ at $\xi$ are continuous in nontrivial transitions; i.e.

$$
\Delta y^{(j+2)}(\xi)=0, \quad j=0,1, \cdots r .
$$

Two cases are distinguished:

(1) The ambiguous case $r<\infty$. Here the Taylor expansions of $\phi(x)$ and $\lambda(x)$ do not exist, and the signs of $\phi(\xi+\varepsilon)$ must be determined by a direct solution of (3).

(2) The degenerate case $r=\infty$. Here the Taylor expansions are identically zero, with $\phi \equiv 0$ and $\lambda \equiv 0$; the two continuations are not distinguishable, as $\phi=0$ is an integral of the Euler equation.

APPENDIX 2. A control problem of the Mayer type is governed by

$$
\begin{aligned}
& y^{\prime}=g(x, y, u), \\
& \phi(x, y, u) \geqq 0,
\end{aligned}
$$

where the control variable $u$ may be absent in $\phi$. The transformation $u=v^{\prime}$ converts the problem into the standard form of the calculus of variations, with the set $\left(y^{\prime}, v^{\prime}\right)$, i.e. $\left(y^{\prime}, u\right)$, playing the role of the slope functions of the field theory. Accordingly, the function $F$ defined by

$$
F=\lambda \cdot\left(-y^{\prime}+g\right)+\mu \phi, \quad \mu \phi=0
$$

must satisfy the Euler equations. As before, two cases are distinguished: case (a) $\phi=\phi(x, y)$, and case (b) $\phi=\phi(x, y, u)$, with the corresponding Hilbert determinants

$$
\begin{aligned}
& H=\left|F_{u u}\right| \phi \\
& H=\left|F_{u u}\right| \phi-\mu \phi_{u} \cdot \hat{F}_{u u} \cdot \phi_{u}
\end{aligned}
$$

on the composite arc. These expressions are analogous to (6) and (29) respectively; hence the conclusions of $\S 7$ hold for the control problem under consideration.

\section{ReFERENCES}

1. G. A. Bliss, Lectures in the Calculus of Variations, University of Chicago Press, 1945.

2. Bliss, op. cit., 204.

3. O. Bolza, Lectures on the Calculus of Variations, Chelsea Publishing Co., (1904), 41-43, 148-151.

4. Bolza, op. cit., 151.

5. B. Garfinkel, Minimal Problems in Airplane Performance, QAM July 1951, 159

6. J. P. Mancil, The Minimum of a Definite Integral with respect to Unilateral Variation, Contributions to the Calculus of Variations, University of Chicago, 1933-37.

7. Mancil, op. cit.

8. F. A. Valentine, Problem of Lagrange with Differential Inequalities, (same publication as 6.) 



\section{PACIFIC JOURNAL OF MATHEMATICS}

\section{EDITORS}

\section{H. SAMELSON}

Stanford University

Stanford, California

\section{R. M. BLUMenthaL \\ University of Washington \\ Seattle, Washington 98105}

*J. DugundjI

University of Southern California Los Angeles, California 90007

\section{RICHARD ARENS}

University of California

Los Angeles, California 90024

\section{ASSOCIATE EDITORS}
E. F. BECKENBACH
B. H. NeUManN
F. WOLF
K. YosIDA

\section{SUPPORTING INSTITUTIONS}

\author{
UNIVERSITY OF BRITISH COLUMBIA \\ CALIFORNIA INSTITUTE OF TECHNOLOGY \\ UNIVERSITY OF CALIFORNIA \\ MONTANA STATE UNIVERSITY \\ UNIVERSITY OF NEVADA \\ NEW MEXICO STATE UNIVERSITY \\ OREGON STATE UNIVERSITY \\ UNIVERSITY OF OREGON \\ OSAKA UNIVERSITY \\ UNIVERSITY OF SOUTHERN CALIFORNIA
}

\author{
STANFORD UNIVERSITY \\ UNIVERSITY OF TOKYO \\ UNIVERSITY OF UTAH \\ WASHINGTON STATE UNIVERSITY \\ UNIVERSITY OF WASHINGTON \\ AMERICAN MATHEMATICAL SOCIETY \\ CHEVRON RESEARCH CORPORATION \\ TRW SYSTEMS \\ NAVAL ORDNANCE TEST STATION
}

Mathematical papers intended for publication in the Pacific Journal of Mathematics should be typewritten (double spaced). The first paragraph or two must be capable of being used separately as a synopsis of the entire paper. It should not contain references to the bibliography. Manuscripts may be sent to any one of the four editors. All other communications to the editors should be addressed to the managing editor, Richard Arens at the University of California, Los Angeles, California 90024.

50 reprints per author of each article are furnished free of charge; additional copies may be obtained at cost in multiples of 50 .

The Pacific Journal of Mathematics is published monthly. Effective with Volume 16 the price per volume (3 numbers) is $\$ 8.00$; single issues, $\$ 3.00$. Special price for current issues to individual faculty members of supporting institutions and to individual members of the American Mathematical Society: $\$ 4.00$ per volume; single issues $\$ 1.50$. Back numbers are available.

Subscriptions, orders for back numbers, and changes of address should be sent to Pacific Journal of Mathematics, 103 Highland Boulevard, Berkeley 8, California.

Printed at Kokusai Bunken Insatsusha (International Academic Printing Co., Ltd.), No. 6, 2-chome, Fujimi-cho, Chiyoda-ku, Tokyo, Japan.

PUBLISHED BY PACIFIC JOURNAL OF MATHEMATICS, A NON-PROFIT CORPORATION

The Supporting Institutions listed above contribute to the cost of publication of this Journal, but they are not owners or publishers and have no responsibility for its content or policies.

* Paul A. White, Acting Editor until J. Dugundji returns. 


\section{Pacific Journal of Mathematics}

\section{Vol. 16, No. 2 December, 1966}

Loren N. Argabright, Invariant means on topological semigroups ........ 193

William Arveson, A theorem on the action of abelian unitary groups ...... 205

John Spurgeon Bradley, Adjoint quasi-differential operators of Euler

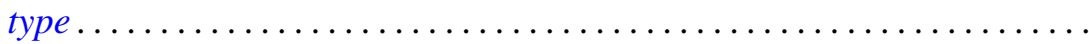

Don Deckard and Lincoln Kearney Durst, Unique factorization in power series rings and semigroups ........................... 239

Allen Devinatz, The deficiency index of ordinary self-adjoint differential operators..................................... 243

Robert E. Edwards, Operators commuting with translations ............ 259

Avner Friedman, Differentiability of solutions of ordinary differential equations in Hilbert space .............................. 267

Boris Garfinkel and Gregory Thomas McAllister, Jr., Singularities in a variational problem with an inequality ......................

Seymour Ginsburg and Edwin Spanier, Semigroups, Presburger formulas,

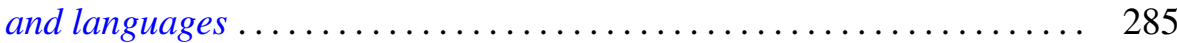

Burrell Washington Helton, Integral equations and product integrals . . . . . . 297

Edgar J. Howard, First and second category Abelian groups with the n-adic topology.........................................

Arthur H. Kruse and Paul William Liebnitz, Jr., An application of a family homotopy extension theorem to ANR spaces.

Albert Marden, I. Richards and Burton Rodin, On the regions bounded by homotopic curves

Willard Miller, Jr., A branching law for the symplectic groups ...

Marc Aristide Rieffel, A characterization of the group algebras of the finite

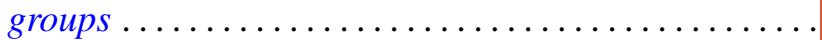

P. P. Saworotnow, On two-sided $H^{*}$-algebras

John Griggs Thompson, Factorizations of p-solvable groups ...

Shih-hsiung Tung, Harnack's inequalities on the classical Cartan

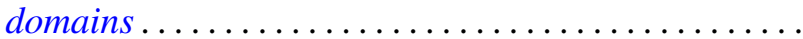

